\title{
Comparison of Different Rainfall Erosion Estimation Methods for the Island of Crete ${ }^{+}$
}

\author{
Dimitrios D. Alexakis ${ }^{1,2, *}$ and Manolis Grillakis ${ }^{1,2}$ \\ 1 School of Environmental Engineering, Technical University of Crete, Chania 73100, Greece; \\ grillakis@hydrogaia.gr \\ 2 Lab of Geophysical-Remote Sensing \& Archaeoenvironment (GeoSat ReSeArch), Institute for \\ Mediterranean Studies, Foundation for Research and Technology Hellas (FORTH), \\ Rethymno 74100, Greece \\ * Correspondence: dalexakis@ims.forth.gr \\ † Presented at TERRAenVISION 2019, Barcelona, Spain, 2-7 September 2019. \\ Published: 21 May 2020
}

\begin{abstract}
Interactions between soil and rainfall plays a vital role in ecological, hydrological and biogeochemical cycles of land. Among those interactions, the phenomenon of rainfall induced soil erosion is crucial to the soil functions, as it affects the soil structure and organic matter content that subsequently affects soil ability to hold moisture and nutrients. The erosive power of a specific rainfall event is regulated by its intensity and total duration. Various methodologies have been developed and tested to estimate the rainfall erosivity in different hydroclimatic regions and using different rainfall measuring timescales. Studies have shown that high temporal resolution measurements provide a more robust erosivity estimation. Nonetheless the sparsity and scarcity of such high temporal resolution data make the accurate estimation of rainfall erosivity difficult. Here, we compare different erosion power estimation methods based on different rainfall timescales for the island of Crete. Sub-daily (30-min) rainfall data based estimation is used as the basis for the assessment of a daily data based estimation methodology and two different methods that use monthly rainfall data. Modified Fournier Index (MFI) is incorporated in the study through different literature approaches and a regression equation is developed between rainfall erosivity power and MFI index for Crete. Results indicate that the use of daily data in the rainfall erosive power estimation is a good approximation of the sub-daily estimation, while formulas based on monthly rainfall data tend to exhibit larger deviations.
\end{abstract}

Keywords: rainfall erosion power; crete; rainfall temporal resolution

Acknowledgments: This research has received funding from the Hellenic Foundation for Research and Innovation (HFRI) and the General Secretariat for Research and Technology Hellas under agreement No 651.

(C) 2020 by the authors. Licensee MDPI, Basel, Switzerland. This article is an open access article distributed under the terms and conditions of the Creative Commons Attribution (CC BY) license (http://creativecommons.org/licenses/by/4.0/). 